\title{
Efficacy and safety evaluation of a hypertonic seawater solution enriched with manganese and copper salts*
}

\author{
Samuel Constant ${ }^{1}$, Amina Saaid², Marcos Alejandro Jimenez-Chobillon ${ }^{3}$ \\ Epithelix, 18 chemin des Aulx CH-1228 Plan-Les-Ouates, Geneva, Switzerland \\ 2Laboratoire Fumouze, 110-114 rue Victor Hugo, 92686, Levallois-Perret, France \\ 3 National Institute of Respiratory Diseases "Ismael Cosío Villegas", México DF, Mexico
}

Rhinology Online, Vol 4: $113-119,2021$

http://doi.org/10.4193/RHINOL/21.021

*Received for publication:

May 7, 2021

Accepted: June 2, 2021

Published: July 9, 2021

\begin{abstract}
Background: Nasal irrigation is commonly recommended as an adjuvant treatment for blocked nose. In the present study, the safety and efficacy profile of Stérimar Blocked Nose (SBN), a hypertonic seawater solution enriched with manganese and copper salts, has been evaluated on nasal epithelium, in vitro.
\end{abstract}

Methodology: 3D reconstituted human nasal epithelium tissue model, MucilAir ${ }^{\mathrm{TM}}$, has been used to investigate the safety of SBN on nasal epithelium by measuring trans-epithelial electrical resistance (TEER), cytotoxicity (lactate dehydrogenase (LDH) release) and phlogosis-related effects (interleukin-8 secretion). Efficacy assessment was measured by ciliary beat frequency (CBF), mucociliary clearance (MCC) and antimicrobial activities (against Staphylococcus aureus and Pseudomonas aeruginosa).

Results: Four-day SBN treatment did not compromise the nasal epithelium integrity as TEER values were over the tissue integrity limit. SBN treatment did not exert cytotoxic (LDH release) or pro-inflammatory effects (IL-8 secretion). SBN treatment significantly increased the CBF and MCC rates compared to untreated cells. SBN treatment exerted a bactericidal effect on $\mathrm{S}$. aureus and $\mathrm{P}$. aeruginosa cultures, whereas seawater not enriched in copper and manganese had only a bacteriostatic effect.

Conclusions: The results demonstrate that SBN is a safe formula for use on human nasal epithelium. The results also suggest a better potential therapeutic role for SBN in comparison to not-enriched seawater when used to control nasal congestion and inhibit bacterial growth which may cause nasal congestion.

Key words: nasal hygiene; hypertonic seawater; nasal irrigation; nasal detersion; nasal congestion: rhinosinusitis.

\section{Introduction}

Nasal congestion is a common complaint in clinical practice and can be associated with a variety of underlying conditions, including rhinitis and rhinosinusitis ${ }^{(1)}$. It has been reported that one in six adults suffers from rhinosinusitis (it is also estimated that this number could be higher since almost $20 \%$ of the affected people does not seek medical care) ${ }^{(2)}$, and nasal congestion is reported by up to $70 \%$ of rhinosinusitis patients ${ }^{(3-5)}$. Although many cases of nasal congestion resolve spontaneously with time, it can be a very uncomfortable symptom and can interfere with daily activities and emotional functioning of the patients due to diminished quality of sleep and increased fatigue, the- reby negatively affecting the quality of life in general $(6,7)$. Specific and universally effective treatments for nasal congestion without adverse effects are not available and symptomatic therapy is the main option ${ }^{(8)}$. Nasal irrigation, also known as nasal douche or nasal wash, is a procedure in which the nasal cavity is rinsed with isotonic or hypertonic saline solutions, and is recommended as one of the adjuvant treatments in a variety of conditions associated with nasal congestion (9-11).

Randomized controlled clinical trials have shown that nasal irrigation with isotonic or hypertonic saline has a beneficial effect on the reduction of nasal congestion associated with chronic rhinosinusitis and quality of life of patients ${ }^{(9,12,13)}$. Isotonic 
solutions are usually recommended for regular application for nasal hygiene, while hypertonic ones are used for decongestion ${ }^{(14)}$. It has also been reported that the use of hypertonic washes could reduce the use of nasal decongestant medications and therefore decrease the risk of side effects due to the overstimulation of adrenergic receptors in the nasal mucosa with potential rebound obstruction ${ }^{(14,15)}$.

Seawater contains many essential minerals such as sodium, bicarbonates, potassium, calcium and magnesium ${ }^{(16)}$. Data from in vitro studies have suggested that calcium and potassium could exert a beneficial effect by stimulating ciliary beat frequency (CBF) and the mucociliary clearance (MCC), an anti-inflammatory response, attributing an advantage of seawater over pure saline solutions without added minerals ${ }^{(13,16)}$. Furthermore, hypertonic seawater solution has been shown to be better than isotonic seawater in the improvement of symptoms of nasal congestion, rhinorrhea, cough, headache and waking up during night ${ }^{(15)}$. Given that hypertonic seawater solutions are used in clinic, the assessment of their safety and efficacy is particularly important. In this paper, a set of in vitro experiments performed to evaluate the safety and efficacy of Stérimar Blocked Nose (SBN), a hypertonic solution $(2.3 \% \mathrm{NaCl})$ composed of filtered seawater enriched with manganese and copper salts, is presented. The safety assessment was made by evaluation of tissue integrity, cytotoxicity and pro-inflammatory profile; and the efficacy assessment was made by measuring mucociliary clearance and bactericidal properties.

\section{Materials and methods}

\section{Test product and the biological model}

SBN is a microfiltered hypertonic seawater solution composed of $75 \%$ seawater (derived from Bay of Cancale, Brittany, France) enriched with copper and manganese salts.

Assays (except the antibacterial activity assessment) were performed in a 3D reconstituted human nasal epithelium model (MucilAir ${ }^{\mathrm{TM}}$, Epithelix Sàrl, Geneva, Switzerland). The human airway epithelia used in this study were reconstituted from primary human cells collected upon surgical nasal polypectomy. All experimental procedures were explained in full, and all subjects provided written informed consent. The study was conducted according to the Declaration of Helsinki on biomedical research (Hong Kong amendment, 1989), and the research protocol was approved by the local ethics committee.

Cells were cultured at the air-liquid interface in $500 \mu \mathrm{l}$ of MuciIAir ${ }^{\mathrm{TM}}$ culture medium in a $\mathrm{CO} 2$ incubator $\left(37^{\circ} \mathrm{C}, 5 \% \mathrm{CO}_{2}, 100 \%\right.$ humidity, Heracell, Waltham, MA, USA) in 24-well plates with 6.5-mm Transwell ${ }^{\circledR}$ inserts (Corning Life Sciences, Corning, NY, USA). Before treatment, inserts were washed with $200 \mu$ l of MuciIAir $^{\mathrm{TM}}$ culture medium and the quality of the tissue was assessed under an inverted microscope (Zeiss Axiovert 25, Oberkochen, Germany).

\section{Treatments}

For TEER, IL-8, LDH and MCC assays Mucilair ${ }^{\mathrm{TM}}$ tissues were left untreated or were treated with $10 \mu \mathrm{l}$ saline $(0.9 \% \mathrm{NaCl})$ or SBN twice a day with an 8-hour interval, for 4 days. SBN was applied on the apical side of the epithelium in 24-well plates. Each day, culture medium was frozen at $-80^{\circ} \mathrm{C}$ for further analysis. The details of each experiment are described below.

Trans-Epithelial Electrical Resistance (TEER): In order to study the tissue integrity by TEER, tissues were left untreated $(n=3)$ or treated with saline $(n=3)$ or SBN $(n=3)$ for 4 days. For this purpose, $10 \mu \mathrm{l}$ of SBN were added to each tissue placed in $700 \mu \mathrm{l}$ of saline solution in 24-well plates. Measurements were performed using a Millicell ERS voltohmmeter (Millipore, Burlington, MA, USA). Three measurements were performed per sample. Basal value was the measurement performed at Day 0 . The resistance of the tissue was calculated by subtracting the blank resistance (insert with no tissue) from the read-out resistance (mean of three) and multiplying by the epithelium surface size $(0.33 \mathrm{~cm} 2)$.

Lactate Dehydrogenase (LDH) secretion: Cytotoxicity was evaluated by quantification of LDH released by dead cells. LDH measurement was performed in untreated $(n=3)$, saline-treated $(n=3)$, SBN-treated $(n=3)$ and $1 \%$ Triton $X-100$ in saline solution $(n=3$, $0.9 \% \mathrm{NaCl}, 1.25 \mathrm{mM} \mathrm{CaCl} 2,10 \mathrm{mM}$ HEPES, Eurospital)-treated tissues (positive control, Fluka Biochemika, $\mathrm{n}=1$ ) at days 1,2,3 and 4 by Cytotoxicity Detection KitPLUS (LDH, Roche, St. Louis, MI, USA) following manufacturer's instructions.

Interleukin-8 (IL-8) secretion: IL-8 secretion evaluation was performed by ELISA (BD OptEIA ${ }^{\mathrm{TM}}$, BD Bioscience, Franklin Lakes, NJ, USA) in untreated $(n=3)$, saline-treated $(n=3), S B N$-treated $(n=3)$ and Cytomix-treated (positive control, $n=3$ ) tissues. Assessment was done at days 1, 2, 3 and 4. Cytomix was composed of $1 \%$ FCS (Amimed, Cat 2-01F36-I, BioConcept Ltd, Allschwil, Switzerland), $0.2 \mathrm{mg} / \mathrm{ml}$ LPS (Sigma) and 500ng/ml TNF-a (GeneTex, Irvine, CA, USA).

Ciliary beat frequency: CBF was measured in untreated $(n=3)$, saline-treated $(n=3), S B N$-treated $(n=3)$ and $50 \mu M$ isoproterenol-treated (positive control, $\mathrm{n}=3$ ) tissues by a special camera system (Sony XCD V60 Firewire) at room temperature and was expressed as $\mathrm{Hz}$. For this purpose, 256 images were recorded for each replicate and CBF was calculated using ciliaFA software.

Mucociliary clearance: Microbeads ( $5 \mu \mathrm{m}$ ) were added onto the apical surface of untreated $(n=3)$, saline-treated $(n=3), S B N-$ treated $(n=3)$ and $50 \mu \mathrm{M}$ isoproterenol-treated (positive control, $\mathrm{n}=3$ ) tissues. For bead tracking, one-minute videos (images taken every second) were recorded using DMIRE2 microscope (Leica, Wetzlar, Germany) equipped with DS-5MC camera (Nikon, 


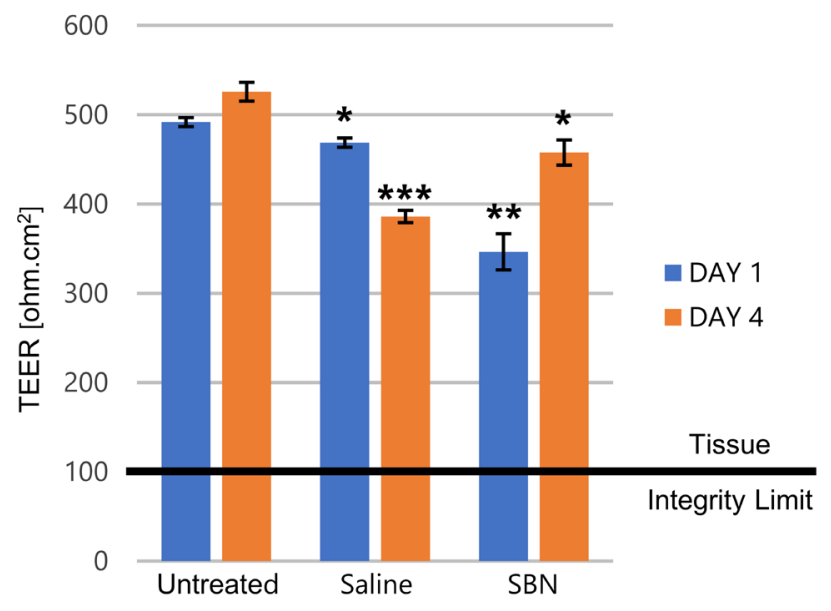

Figure 1. Effect of SBN treatment on tissue integrity monitored by TEER on untreated $(n=3)$, saline-treated $(n=3), S B N$ - treated $(n=3)$ tissues at Day 1 and Day 4 post-treatment. ${ }^{*} p<0.05,{ }^{* * *} p<0.001$, compared to untreated cultures. Error bars represent SEM.

Tokyo, Japan). MCC was measured at days 1 and 4. Results represent an average of 200-500 beads tracked (Image Pro Plus, Media Cybernetics, Rockville, MD, USA).

\section{Assessment of bacterial activity}

Staphylococcus aureus (ATCC 6538) and Pseudomonas aeruginosa (ATCC 9027) were thawed out and subcultured in Tryptic Soy Agar (TSA) plates and then in suspension at $30-35^{\circ} \mathrm{C}$ up to $10^{8}$ CFUs (as calculated by OD620). SBN solution (1\% final treatment concentration) was added to the suspension to ensure minimal changes in growth characteristics. Monitoring was carried out at a 24-hour interval with counts immediately after (0h) and 1, 3 and 24 hours after the treatment. After appropriate dilutions, $S$. aureus and $P$. aeruginosa were plated on TSA plates. Counting was performed on TSA plates after incubation at $30-35^{\circ} \mathrm{C}$. TSA plates without addition of SBN were used as growth control. The effect of SBN was also compared to that of isotonic $(0.9 \% \mathrm{NaCl})$ and hypertonic $(2.2 \% \mathrm{NaCl})$ seawater controls which were not enriched with copper and manganese.

\section{Statistical analyses}

Statistical analyses were performed by T-test (two-tailed) by Microsoft Excel.

\section{Results}

Evaluation of tissue integrity

In tissues treated with SBN, a significant decrease in TEER was observed at both Day 1 and Day 4 after treatment (Figure 1). Similarly, significant decreases were observed in saline treated cultures. Nevertheless, these values were above the range of an intact epithelium $\left(>100 \Omega . \mathrm{cm}^{2}\right)$, indicating that SBN treatment does not compromise epithelial integrity.

\section{Lactate dehydrogenase secretion}

As seen in Figure 2, tissues treated with SBN and saline had a similar LDH profile to that of untreated cells, indicating that these solutions are not cytotoxic. Treatment with Triton X-100 was used as positive control for cell lysis corresponding to $100 \%$ cytotoxicity.

\section{Evaluation of pro-inflammatory profile}

As observed in Figure 3, SBN did not induce pro-inflammatory effects. In fact, a significant decrease in the release of IL-8 occurred after the application of SBN in comparison with untreated cultures $(p=0.0009, p=0.0016$, and $p=0.0005$ for Days 2,3 and 4 , respectively), which confirmed that SBN is not a trigger of inflammation. In saline-treated tissues, a significant increase in IL-8 release was observed on Day $1(p=0.0217)$, which was not observed at later time points. Cytomix was used as a positive control, and, as expected, it induced a significant release of IL-8 at all timepoints.

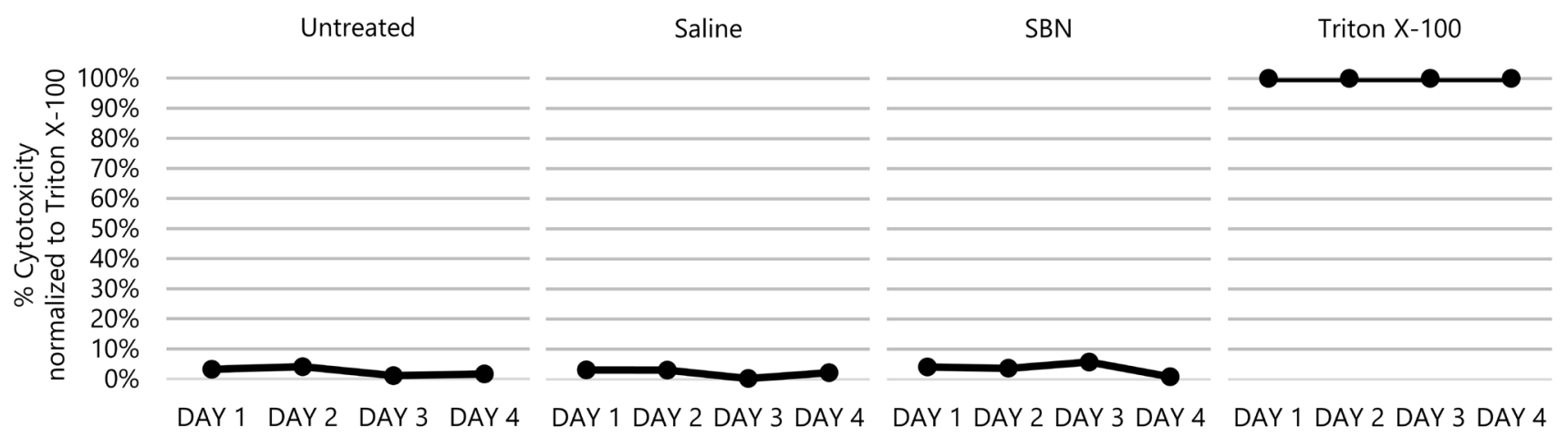

Figure 2. Effect of SBN on cytotoxicity. Secreted lactate dehydrogenase (LDH) profile after treatment with SBN. Levels of LDH were measured in untreated $(n=3)$, saline-treated $(n=3)$, SBN-treated $(n=3)$ and Triton X-100-treated ( $n=3)$ MucilAir ${ }^{T M}$ epithelial cells for 1-4 days. Cytotoxicity was analyzed at days 1, 2, 3 and 4 by monitoring $\mathrm{LDH}$ release. 


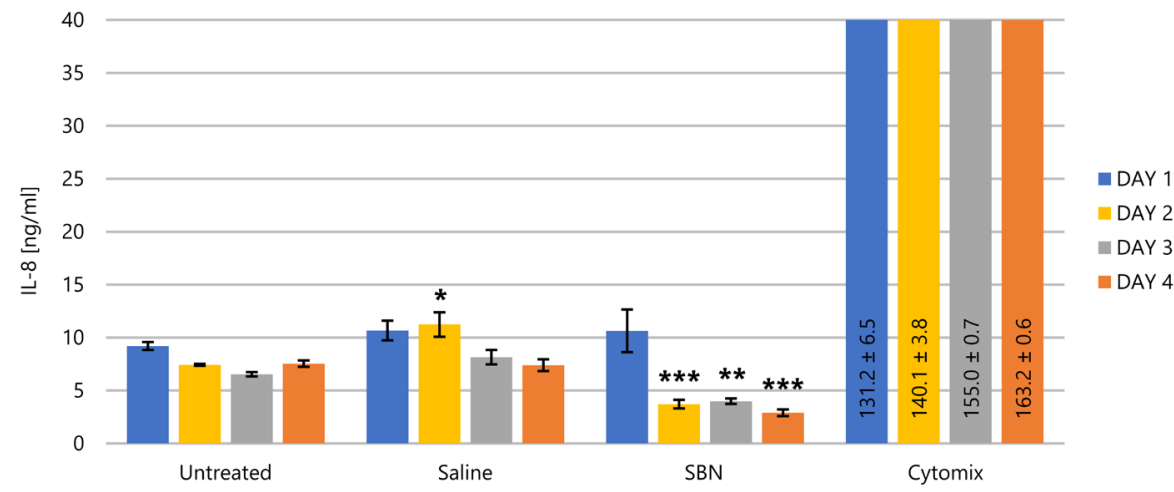

Figure 3. Effect of SBN on IL-8 secretion. IL-8 levels were measured in an ELISA assay in MucilAir ${ }^{\mathrm{TM}}$ epithelium of untreated $(n=3)$, saline-treated $(n=3)$, SBNtreated $(n=3)$ and Cytomix-treated $(n=3)$ tissues for 4 days. IL-8 was monitored at days $1,2,3$ and $4 ;{ }^{*} p<0.01,{ }^{* *} p<0.01$ and *** $p<0.001$ compared to untreated cultures. Error bars represent SEM.

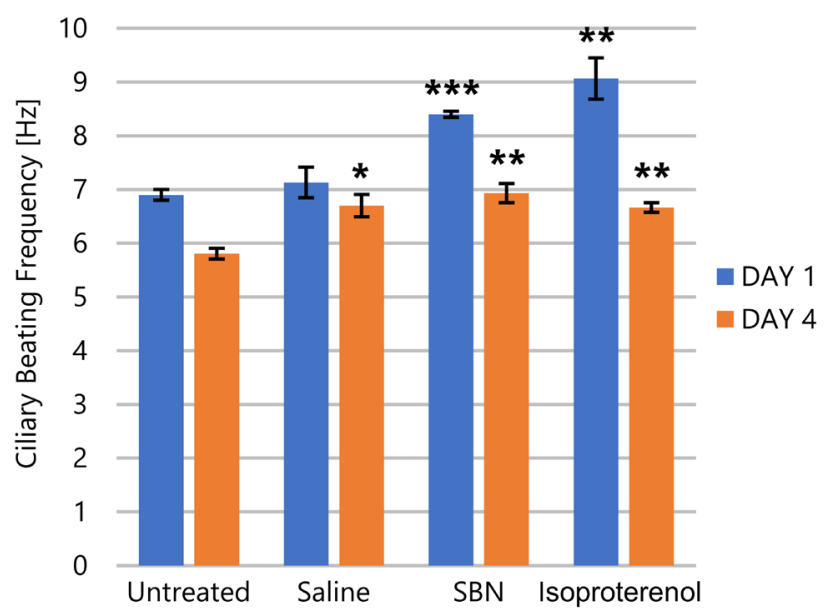

Figure 4. Effect of SBN on ciliary beat frequency. Untreated $(n=3)$, salinetreated $(n=3)$, SBN-treated $(n=3)$ and isoproterenol-treated $(n=3)$ tissues were monitored on days 1 and $4 ;{ }^{*} p<0.01,{ }^{* *} p<0.01$ and ${ }^{* * *} p<0.001$ compared to untreated cultures. Error bars represent SEM.

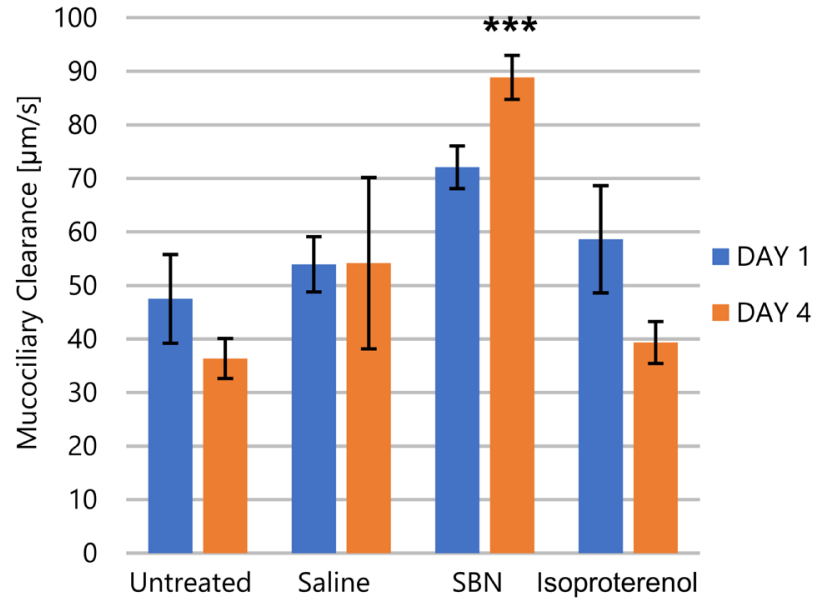

Figure 5. Effect of $S B N$ on mucociliary clearance. Untreated $(n=3)$, salinetreated $(n=3)$, SBN-treated $(n=3)$ and isoproterenol- treated $(n=3)$ tissues were monitored on days 1 and $4 ;{ }^{* * *} \mathrm{p}<0.001$ compared to untreated cultures. Error bars represent SEM.

\section{Ciliary Beat Frequency (CBF)}

Treatment with SBN significantly increased CBF at Day 1 and Day 4 when compared to untreated cells (Day $1: 6.9 \mathrm{~Hz}$ vs $8.4 \mathrm{~Hz}$, $\mathrm{p}=0.0002$; Day 4: $5.8 \mathrm{~Hz}$ vs $6.9 \mathrm{~Hz}, \mathrm{p}=0.0051$ ) (Figure 4). A significant but milder increase has been observed in saline-treated tissues on Day 4 compared to untreated tissues $(p=0.018)$.

\section{Mucociliary clearance (MCC)}

Treatment with SBN significantly increased the microbead clearance velocity at Day 4 when compared to untreated cells (36.4 $\mu \mathrm{m} / \mathrm{s}$ vs $88.9 \mu \mathrm{m} / \mathrm{s}, \mathrm{p}=0.0007$ ) (Figure 5). No significant changes were observed in saline-treated tissues compared to untreated tissues. The MCC rate of SBN-treated cells was even higher than the rate observed in tissues treated with isoproterenol (positive control) at Day $4(\mathrm{p}=0.0001)$.

\section{Antimicrobial activity}

SBN exerted a bactericidal effect on the growth of S. aureus and $P$. aeruginosa starting three hours and one hour after application, respectively (Figure 6). On the contrary, in isotonic and hypertonic seawater-treated samples, bacterial growth was inhibited, and this effect persisted until the end of the experiment $24 \mathrm{~h}$ after application indicating a bacteriostatic effect of these solutions.

\section{Discussion}

Nasal congestion is one of the most commonly reported symptoms in medical practice and it can be associated with a variety of conditions, with rhinitis, rhinosinusitis and allergic rhinitis being the most frequent ones ${ }^{(1)}$. As an adjuvant treatment, nasal irrigation is frequently used to reduce nasal congestion (9-11). Hypertonic seawater solutions have been proven to be the most effective non-pharmacological treatment to decrease the symptoms of nasal congestion most likely due to the draining of excess water from cells by inducing osmosis ${ }^{(15)}$.

In the present study, in vitro evidence on the safety and efficacy of SBN, a hypertonic seawater solution enriched with manganese and copper salts has been presented. The majority of in vitro assays were performed in a MucilAir ${ }^{\mathrm{TM}}$ 3D model which simulates the morphological and metabolic characteristics of the nasal 

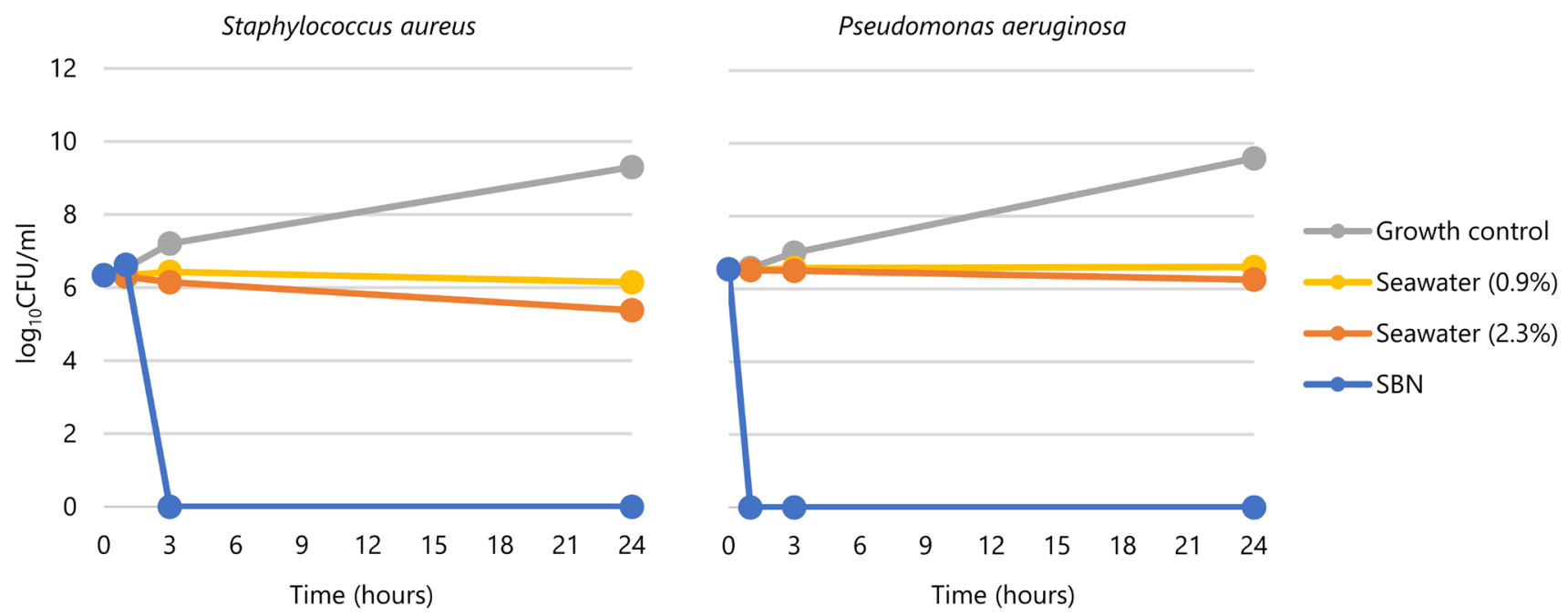

Figure 6. Efficacy of SBN against S. aureus and P. aeruginosa. Growth of germs in bacterial cultures treated with TSA (control), TSA + isotonic seawater (0.9\%), TSA + hypertonic seawater (2.3\%), TSA + SBN.

epithelium in vivo and is increasingly been used for the safety evaluation of substances ${ }^{(17-19)}$.

The safety of the product was first tested by evaluating the integrity of the epithelium tissue using a TEER assay after an apical exposure to the test formulation. This technique evaluates the stability of tight junctions in the epithelium and is therefore used as a measure of the barrier function in cell culture models of endothelial and epithelial monolayers. It is the measurement of electrical resistance across a cellular monolayer and it is accepted as a very sensitive and reliable method to confirm the integrity and permeability of the monolayer ${ }^{(20)}$. Maintenance of stability and electrical resistance of the epithelium is of crucial importance for essential physiological processes. Thus, significant changes in TEER may be an early sign of cell damage (21). Although TEER values were lower upon treatment with SBN when compared to untreated cells, they remained within the ranges of an intact epithelium. Moreover, similar decrease has been observed with saline treatment.

Apart from serving as a physical barrier, the airway epithelium also functions as a modulator of the immune response by release of a number of cytokines, especially IL-8 ${ }^{(22,23)}$. Quantification of IL-8 after application of SBN revealed that there was no induction of inflammation (indeed there were statistically significant decreases on Days 2, 3 and 4). The cytotoxicity of the tested formula was also evaluated in the Mucil/Air ${ }^{\mathrm{TM}} 3 \mathrm{D}$ model by quantifying the $L D H$ released by dead cells ${ }^{(24)}$. Similar results were obtained for untreated cultures and the cultures treated with saline or SBN, demonstrating that SBN is not cytotoxic. These results indicate that the application of the hypertonic seawater solution on nasal epithelium is safe. After concluding the safety profile of the tested formula, the efficacy of SBN was evaluated through a series of in vitro assays.

It has been suggested that nasal saline irrigation could exert its beneficial effects by removing antigens and irritant particles through improved MCC which is a first line defense mechanism that aids the airway epithelia to clear foreign particles and chemicals, contributing to better respiratory function ${ }^{(25,26)}$. MCC is widely dependent on $C B F$, and CBF is partly linked to MCC rates. The analysis of the results on the efficacy of SBN on MCC revealed that the MCC significantly increased upon treatment with SBN at day 4 in comparison to untreated cultures. MCC velocity of SBN treated cultures was even higher than the positive control isoproterenol, a bronchodilator known to activate CBF (27). In fact, our data shows that CBF was significantly increased in SBN-treated tissues compared to untreated-tissues. In clinical settings, this increase in MCC and CBF rates may result in a higher and quicker removal of debris, virus, bacteria and potential irritants from the nasal cavity lining.

As part of the in vitro evaluation of its efficacy, the antibacterial activity of SBN was assessed against $S$. aureus (Gram-positive) and $P$. aeruginosa (Gram-negative) species, two bacteria known to colonize upper airway cells in some circumstances ${ }^{(28,29)}$. Results showed that SBN has a bactericidal effect against these bacteria starting as early as 1 hour for $P$. aeruginosa and 3 hours for $S$. aureus, and this effect lasted until the end of the experiment (24h). In contrast, both isotonic and hypertonic seawater not enriched in copper and manganese had only a bacteriostatic effect; therefore, the bactericidal effects of SBN may be attributed to the presence of copper and/or manganese in its formulation. Copper and manganese are found in very small amounts in the human body. They are reported to stimulate the body's self-defense mechanisms ${ }^{(30,31)}$. Copper salts may aid in fighting viral infections as well, as cupric ions have been shown to inactivate several types of viruses, including members of the herpesvirus and arenavirus families ${ }^{(28)}$. In addition, it has been shown that copper salts have great potential to be used to 
control the growth of both gram positive, gram negative, and multi-drug resistant bacteria isolated from clinical samples ${ }^{(32,33)}$. It has been reported that cations such as copper and silver are electrostatically attracted to heterotrophic bacteria which have a net negative surface charge at around neutral $\mathrm{pH}$ values. This attraction results in the induction of cell ionization and bacterial inactivation. The copper-induced bacterial inactivation is related to its oxidation power through electrolytically formed hydrogen peroxide. Moreover, copper has been shown to facilitate hydrolysis and nucleophilic displacement reactions which has been shown to disrupt several cell enzymatic pathways and break hydrogen bonds, thus directly causing DNA damage ${ }^{(34)}$. The limitations of the study include the low number of replicates used in the assays and the controls used in different experiments: ie, saline in TEER, LDH, IL-8, CBF and MCC, and isotonic and hypertonic seawater solutions in antibacterial assays. Considering the positive results obtained in this study, further tests with a larger number of samples and consistent inter-experiment controls should be performed in the future.

\section{Conclusions}

As a summary, in this study, SBN has been shown to be safe for use on human nasal epithelium. The results also suggest a better potential therapeutic role for SBN in comparison to not-enriched seawater when used to control nasal congestion, as shown by its efficacy on stimulating CBF and MCC, and on inhibiting bacterial growth which may cause nasal congestion. However, clinical trials are warranted.

\section{Authorship contribution}

SC, AS, and MAJC established the study conception and design. SC, AS, and MAJC developed the experimental methodology. SC, AS, and MAJC acquired, analysed and interpreted data. SC, AS, and MAJC wrote, reviewed and revised the manuscript. SC, AS, and MAJC supervised the study.

\section{Acknowledgments}

Authors would like to thank Dr. Philippe Contencin and Dr. Annahita Ghassemi for their valuable contribution to the elaboration and revision of the manuscript.

\section{Funding}

This study has been sponsored by Church \& Dwight, Co., Inc.

\section{Ethics approval and consent to participate}

Not applicable.

\section{Consent for publication}

Not applicable.

\section{Availability of data and materials}

Not applicable.

\section{Conflict of interest}

AS formerly worked as EU Technology \& Innovation Manager at Church \& Dwight, Co., Inc. SC and MAJC declare no conflict of interest in this work.

\section{References}

1. Krouse J, Lund V, Fokkens W, Meltzer EO Diagnostic strategies in nasal congestion. Int J Gen Med. 2010;3:59.

2. Leggett JE. Acute sinusitis: Whenand when not-to prescribe antibiotics. Postgrad Med. 2004;115(1):13-9.

3. Stewart M, Ferguson BJ, Fromer L Epidemiology and burden of nasal congestion. Int J Gen Med. 2010;3:37.

4. Ferrand PA, Mercier CH, Jankowski R, et al. Acute sinusitis in adults. Management by general practitioners. Presse med. 2001;30(21):1049-54.

5. Pessey JJ, Reitz C, Los F. Acute rhinosinusitis in the adult: national survey of general practice management. Rev Laryngol Otol Rhinol (Bord). 2000;121(4):237-41.

6. Shedden A. Impact of nasal congestion on quality of life and work productivity in allergic rhinitis. Treat Respir Med. 2005;4(6):43946.

7. Goetzel RZ, Hawkins K, Ozminkowski RJ, Wang S. The health and productivity cost burden of the "top 10" physical and mental health conditions affecting six large US employers in 1999. J Occup Environ Med.
2003;45(1):5-14

8. Deckx L, De Sutter Al, Guo L, Mir NA, van Driel ML. Nasal decongestants in monotherapy for the common cold. Cochrane Database Syst Rev. 2016(10)

9. Fokkens WJ, Lund VJ, Hopkins C, et al European position paper on rhinosinusitis and nasal polyps 2020. Rhinol. 2020;58(Suppl S29):1-464

10. Price D, Bond C, Bouchard J, et al. International Primary Care Respiratory Group (IPCRG) guidelines: management of allergic rhinitis. Prim Care Respir J. 2006;15(1):58-70

11. Wei CC, Adappa ND, Cohen NA. Use of topical nasal therapies in the management of chronic rhinosinusitis. Laryngoscope. 2013;123(10):2347-59.

12. Talbot AR, Herr TM, Parsons DS. Mucociliary clearance and buffered hypertonic saline solution. Laryngoscope. 1997;107(4):500-3.

13. Friedman $M$, Vidyasagar $R$, Joseph $N$. A randomized, prospective, double-blind study on the efficacy of Dead Sea salt nasal irrigations. Laryngoscope. 2006;1 16(6):878-82.

14. Laberko EL, Bogomil'sky MR, Soldatsky YL, Pogosova IE. The influence of an isotonic solution containing benzalkonium chloride and a hypertonic seawater solution on the function of ciliary epithelium from the nasal cavity in vitro. Vestn Otorinolaringol. 2016;81(2):49-52.

15. Culig J, Leppee M, Vceva A, Djanic D. Efficiency of hypertonic and isotonic seawater solutions in chronic rhinosinusitis. Med Glas. 2010;7(2):116-23.

16. Bastier $P L$, Lechot $A$, Bordenave L, Durand M, De Gabory L. Nasal irrigation: from empiricism to evidence-based medicine. A review. Eur Ann Otorhinolaryngol Head Neck Dis. 2015;132(5):281-5.

17. Reus AA, Maas WJ, Jansen HT, et al. Feasibility of a 3D human airway epithelial model to study respiratory absorption. Toxicol In Vitro. 2014;28(2):258-64.

18. Kuper CF, Gröllers-Mulderij M Maarschalkerweerd T, et al. Toxicity assessment of aggregated/agglomerated cerium oxide nanoparticles in an in vitro 3D airway model: The influence of mucociliary clearance. Toxicol In Vitro. 2015;29(2):389-97.

19. Stewart CE, Torr EE, Mohd Jamili NH, Bosquillon C, Sayers I. Evaluation of differentiated human bronchial epithelial cell cul- 
ture systems for asthma research. J Allergy. 2012;2012.

20. Srinivasan B, Kolli AR, Esch MB, Abaci HE, Shuler ML, Hickman JJ. TEER measurement techniques for in vitro barrier model sys tems. J Lab Autom. 2015;20(2):107-26.

21. Ardolino LI, Meloni M, Brugali G, Corsini E, Galli CL. Preclinical evaluation of tolerability of a selective, bacteriostatic, locally active vaginal formulation. Curr Ther Res Clin Exp. 2016;83:13-21.

22. Vareille $M$, Kieninger $E$, Edwards $M R$, Regamey N. The airway epithelium: soldier in the fight against respiratory viruses. Clin Microbiol Rev. 2011;24(1):210-29.

23. Huibi CA, Anan WA, Martin B, et al. Downregulation of $I L-8$ expression in human airway epithelial cells through helper-dependent adenoviral-mediated RNA interference. Cell Res. 2005;15(2):111-9.

24. Cho MH, Niles A, Huang R, et al. A bioluminescent cytotoxicity assay for assessment of membrane integrity using a proteolytic biomarker. Toxicol In Vitro. 2008;22(4):1099-106.

25. Delmotte P, Sanderson MJ. Ciliary beat frequency is maintained at a maximal rate in the small airways of mouse lung slices. Am Respir Cell Mol Biol. 2006;35(1):110-7.

26. Anselmo-Lima WT, Sakano E, Tamashiro $E_{\text {, }}$ et al. Rhinosinusitis: evidence and experience. A summary. Braz J Otorhinolaryngol.
2015:81(1):8-18.

27. Allen-Gipson DS, Romberger DJ, Forget MA, May $\mathrm{KL}$, Sisson JH, Wyatt TA. IL-8 inhibits isoproterenol-stimulated ciliary beat frequency in bovine bronchial epithelial cells. J Aerosol Med. 2004;17(2):107-15.

28. Pettigrew MM, Gent JF, Revai K, Patel JA, Chonmaitree T. Microbial interactions during upper respiratory tract infections. Emerg Infect Dis. 2008;14(10):1584.

29. Fothergill JL, Neill DR, Loman N, Winstanley C, Kadioglu A. Pseudomonas aeruginosa adaptation in the nasopharyngeal reservoir leads to migration and persistence in the lungs. Nat Commun. 2014;5(1):1-9.

30. Foreman JC, Mongar JL. The action of lanthanum and manganese on anaphylactic histamine secretion. Br J Pharmacol. 1973;48(3):527.

31. Sagripanti $J L$, Routson LB, Bonifacino $A C$, Lytle CD. Mechanism of coppermediated inactivation of herpes simplex virus. Antimicrob Agents Chemother. 1997;41(4):812-7.

32. Febre N, Silva $V$, Baez A, et al. Antibacterial activity of copper salts against microorganisms isolated from chronic infected wounds. Rev Med Chile. 2016;144:1523-30.

33. Benhalima L, Amri S, Bensouilah M, Ouzrout R. Antibacterial effect of copper sulfate against multi-drug resistant nosocomial pathogens isolated from clinical samples. Pak J Med Sci. 2019;35(5):1322.

34. Thurman RB, Gerba CP, Bitton G. The molecular mechanisms of copper and silver ion disinfection of bacteria and viruses. Crit Rev Environ Sci Technol. 1989;18(4):295-315.

Marcos Alejandro Jimenez-Chobillon Department of Otorhinolaryngology and Head and Neck Surgery National Institute of Respiratory Diseases "Ismael Cosío Villegas"

Calz Tlalpan 4502

Col Sección XVITlalpan

CP 14080

México DF

Mexico

Tel: + 52-55-10229830

E-mail: aljicho@gmail.com

ISSN: 2589-5613 / @2021 The Author(s). This work is licensed under a Creative Commons Attribution 4.0 International License. The images or other third party material in this article are included in the article's Creative Commons license, unless indicated otherwise in the credit line; if the material is not included under the Creative Commons license, users will need to obtain permission from the license holder to reproduce the material. To view a copy of this license, visit http://creativecommons.org/ licenses/by/4.0/ 